\title{
Dark Energy: Accelerating Expansion of the Universe and of the Higgs Quantum Space
}

\author{
Jacob Schaf \\ Instituto de Física, Universidade Federal do Rio Grande do Sul (UFRGS), Av. Bento GonÃğalves, 9500, CEP: 91501-970, Porto Alegre-RS, Brazil
}

Copyright (C) 2015 by authors, all rights reserved. Authors agree that this article remains permanently

open access under the terms of the Creative Commons Attribution License 4.0 International License

\begin{abstract}
Dark energy, accelerating the expansion of the universe, is fourteen times larger than the ordinary mass-energy in the universe. So much energy can be found only in the scenario of the creation of the universe. Quantum condensation of the Higgs condensate (HC), after the Big-Bang, when the temperature fell through $10^{15}$ Kelvin, liberates hundreds of $\mathrm{GeV} /$ boson. The Higgs potential energy well has the form: $U(\rho)=-n\left(\phi^{*} \phi\right)+m\left(\phi^{*} \phi\right)^{2}$, where $n>m, \phi$ is a complex order parameter and $\rho=\phi^{*} \phi$ is the condensate density. The depth of this potential well is generic and uniform throughout the universe. If $\rho>n / m$, the $H C$ can lower its energy by freely expanding its volume, which is related with the accelereted expansion of the universe. Recent experimental observations, achieved with the help of the tightly synchronized clocks in orbit, show that the Higgs Quantum Space (HQS), ruling the inertial motion of matter and propagating light, is circulating round earth, round the sun and round the galactic center according to velocity fields, consistent with the local main astronomical motions, thereby appropriately creating the observed gravitational dynamics. In these Keplerian velocity fields, earth is very closely stationary with respect to the HQS, which explains the isotropy of light with respect to earth. It also explains the absence of the gravitational slowing of the GPS clocks, predicted by General Relativity, but not observed. This HQS-dynamics however cannot explain why the recession between the galaxies causes no light anisotropy. The isotropy of light proves that this recession too lets earth, the sun and the Milky-Way galaxy stationary with respect to the HQS. Obviously, this becomes possible only if the expansion of the universe is expansion of the HQS itself, showing that the expansion of the universe follows perfectly the expansion of the HQS itself.
\end{abstract}

Keywords Dark Energy, Accelerated Expansion of the Universe, Higgs Quantum Space, Gravitational Dynamics, Gravitational Effects

\section{Introduction}

Recent astrophysical observations have confirmed that the Universe shows an accelerated cosmic expansion. [1,2] Evidence of this expansion has been shown independently from measurements of supernovae of type Ia and from microwave background radiation $[3,4]$. It has been proposed that this cosmological behavior is caused by a hypothetical dark energy $(w)$, a cosmic fluid parameterized by an equation of state $w=p / \rho$, where $p$ is the spatial homogeneous pressure and $\rho$ is the dark energy density $[3,4,5]$. These models are based in a modified Schwarzschild metric, in which the gravitational time dilation plays an essential role. The problem is that, while atomic clocks, stationary in a gravitational field, are slowed according to the predictions by General Relativity (GR), the atomic clocks in the GPS satellites, moving with earth round the sun, are not slowed by the solar field. $[6,7]$ The orbital motion of earth clearly suppresses the gravitational time dilation, due to the sun. GR relativity cannot explain this absence because the gravitational potential is a scalar. Here in the second paragraph of Section 2.1 details will be given). Estimates by cosmologists also came to the astonishing conclusion that the amount of dark energy, necessary to explain this observed accelerated expansion, is fourteen times larger than the total visible mass-energy in the universe. Obviously, no physical process of ordinary massenergy can provide so much energy. Therefore, the origin of dark energy must be searched in the scenario of the creation of the universe.

The consensus among the cosmologists is that dark energy is not ordinary mass-energy, but is energy of space itself and distributes it uniformly throughout the universe. It also is consensus that dark energy interacts with ordinary mass-energy only by gravity. These conclusions, although perfectly plausible from the viewpoint of the observations, are made without appointing a physical background, able to give rise to these features. The present work will show that all these features arise naturally and precisely within the scenario of the Higgs Quantum Space (HQS). HQS-dynamics is shown in Reference [8] to create the gravitational dynamics throughout the universe. It also appropriately predicts all the observed effects of the gravitational fields on the propagation of light and on the rate of the clocks.

According to the Higgs theory, quantum condensation of 
the Higgs bosons closely after the Big-Bang, when the temperature of the universe fell through $10^{15}$ Kelvin, liberates hundreds of $\mathrm{GeV}$ per Higgs boson. Quantum condensation of bosons takes place because of the Bose-Einstein (BE) phase correlation between the wave functions of the bosons. At sufficiently low temperatures BE-correlation overcomes the thermal fluctuations and the boson system can lower its energy by spontaneously breaking the universal $U(1)$ gauge symmetry of the boson wave functions and condensing all the particles into the same quantum state. Along this transition, the particles assume all the same phase giving rise to a long-range phase coherent macroscopic quantum state, which usually is known as quantum fluid. In the condensate, the particles become totally entangled and indistinguishable.

In the condensation of the usual superfluids and superconducting condensates (SCC), BE correlation is weak and relatively short-range (only tens of nanometers), involving typically millielectron volts $(\mathrm{meV}$ ) of energy per boson. Therefore, condensation occurs only at very low temperatures and the coherence length is relatively short. However, condensation of the Higgs bosons takes place at $10^{15}$ Kelvin and involves hundreds of $\mathrm{GeV}$ per Higgs boson. This evidences that the BE phase correlation between the Higgs bosons is incredibly strong (very long-range), giving rise to a very large phase coherence length of the Higgs condensate (HC).

Cosmologists estimate that the number of Higgs bosons in the universe is larger than that of the hadrons. Hence, the total energy supplied by quantum condensation of the Higgs is largely sufficient to drive the expansion of the universe. This condensation however needs a physical mechanism that can dissipate so much energy. Insufficient dissipation obviously slows down the condensation rate. In particular, the presence of the ordinary mass-energy in the universe represents persistent phase disorder that holds back the advance of the HC toward the minimum of energy. Therefore, the condensation necessarily is gradual. The presence of the ordinary massenergy and the observed evolution of the visible universe give strong evidence that actually this condensation process still is in course.

The quantum condensate is described by a complex macroscopic (Ginsburg-Landau like) order parameter $\phi=$ $\phi(x, y, z) e^{i \theta_{0}}$ that represents the resting condition (ground state) of the condensate. In this expression $\phi(x, y, z)$ is the amplitude of the order parameter as a function of position, $\theta_{0}$ is the phase and $\rho(x, y, z)=\phi^{*} \phi$ is the local volumetric condensate density. This density intrinsically distributes it uniformly throughout the volume of the condensate, which, in the case of the Higgs condensate, covers the whole of space. Interestingly, the complex order parameter allows the condensate to undergo internal motion without any perceptible classical displacement.

The BE quantum phase correlation gives rise to a negative potential energy (bonding) term, the value of which increases linearly with the condensate density $\rho=\phi^{*} \phi$. Another positive potential energy (anti-bonding) term arises from repulsive core interaction between the bosons, that increases with the squared density $\left(\phi^{*} \phi\right)^{2}$ and prevents collapse of the system. The effective potential $U(\rho)$ has the form:

$$
U(\rho)=-n\left(\phi^{*} \phi\right)+m\left(\phi^{*} \phi\right)^{2}
$$

where however the value of the coefficient of the bonding term $n$ is considerably larger than $m$ of the anti-bonding term. Hence, the minimum of the effective potential energy occurs not for $\phi^{*} \phi=0$, as would be usual, however for a finite value $\phi^{*} \phi=n / m$. This is known as a non-zero vacuumexpectation-value. As the phase $\left(\theta_{0}\right)$ can take values within zero and $2 \pi$, the potential energy well is symmetric about $\phi=0$ and thus has the form of a Mexican sombrero in the complex $\phi_{R e}$ and $\phi_{I m}$ plane and rotations in $\theta$ involve no energy (please see Fig. 1).

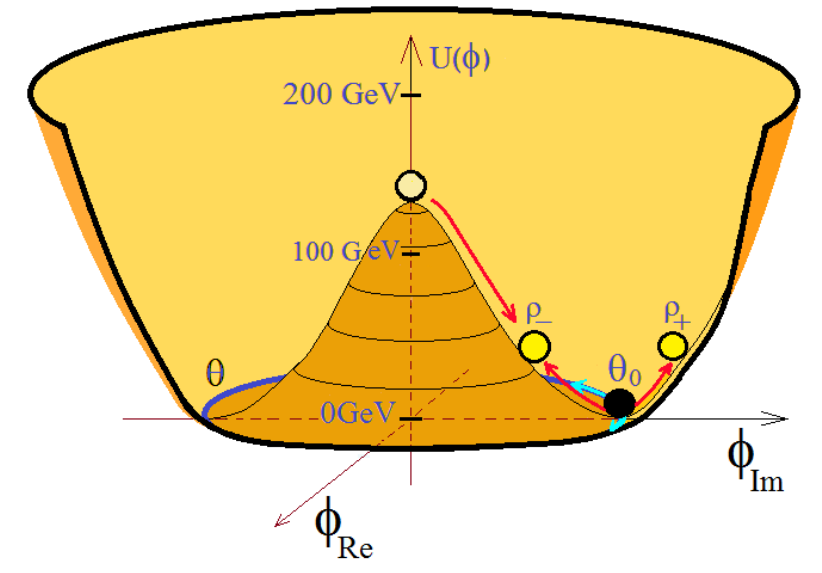

Figure 1. Potential Well, Characteristic of Bose-Einstein Condensates: The figure depicts the form of the Mexican sombrero potential, characteristic of Bose-Einstein condensates (Higgs condensate). The energy scale is per condensed Higgs boson. A red arrow indicates the transition toward the lower energy phase coherent state with the well-defined phase $\theta_{0}$. The figure also indicates the high volumetric density $\left(\rho_{+}\right)$and low volumetric density $\left(\rho_{-}\right)$states. While $\rho_{+}$ drives accelerated expansion, $\rho_{-}$drives accelerated contraction of the volume of the condensate. This is the Higgs mode. The Goldstone mode is indicated along the blue bottom circle.

The deepness of the Mexican sombrero potential well depends on the strength of the BE correlation between the boson wave functions, which in its turn depends on the correlation length. While the deepness in the case of the SCC is in the order of $m e V s$, in the case of the Higgs condensate the deepness is in the order of hundreds of GeVs.

Any local displacement of the phase within a small volume of the condensate, with respect to the overall phase $\left(\theta_{0}\right)$ of the order parameter, costs energy, because it must conquer with the local phase correlation and climb up in the potential energy. This gives rise to a peculiar and well known transient quantum phase stiffness that nevertheless manifests it only during new displacements of the phase and enables the quantum condensates to transmit longitudinal as well as transverse elastic oscillations analogously as elastic solids.

Local phase displacement of the order parameter of a quantum condensate inherently is associated with local flow of the condensate. While a constant phase gradient $(\nabla \theta)$ makes the condensate to flow uniformly without any resistance along the phase gradient with velocity proportional to the magnitude of the gradient, a phase gradient changing with time corresponds to accelerated motion of the condensate. Due to the phase stiffness, such motions, once excited, automatically become persistent or propagate at a characteristic velocity (the velocity of light in the case of the HQS). For instance, if a current is excited in the superconducting coils of a superconducting magnet by an applied electromotive force (electric field), it will flow forever, even after the electromotive force is cut-off and can be stopped only by an opposite electromotive force. This persistence is the origin of the inertial mass of 
photons within superconductors.[9] In the case of the Higgs Quantum Space (HQS), an analogous persistence of the excited motions (flows) gives rise to the mechanical inertia of the particles related with conservation of the linear and angular momenta of matter bodies. Both the SCC and the HC are macroscopic quantum states (quantum fluids), which is the reason of their completely analogous phenomenologies. In fact to each effect in the SCC, there is an analog of the HQS.

$\mathrm{BE}$ condensates couple only to specific fields. It is well known that the SCC interacts only with electromagnetic (EM) fields. Superconductivity and magnetic fields are intrinsically incompatible with each other. The reason is that the vector potential, associated with the magnetic field, causes local phase displacements of the superconducting order parameter, introducing phase disorder and thereby increasing the energy of the system, locally destroying superconductivity and recovering the $U(1 \mid)$ gauge symmetry. Superconductors can lower their energy by confining the magnetic field into quantized microscopic fluxons or developing a macroscopic screening velocity field of the SCC and a Lorentz force field, expelling out the magnetic field. This is the Meissner effect, responsible for the levitation of magnets by superconductors. [10] In non-homogeneous superconductors the superconductor can also reduce its energy compressing the magnetic field into regions where the order parameter is weaker or zero. This gives rise to the high field paramagnetic Meissner effect, [11]. The HC couples only to the weak nuclear field and indirectly to all quarks and leptons containing hypercharges. The fields associated with the elementary particles too cause phase disorder in the Higgs order parameter, increasing the energy of the $\mathrm{HC}$ and impeding it to reach the minimum of energy. The $\mathrm{HC}$ can lower its energy by confining the fields, associated with the elementary particles, to microscopic regions of space $\left(10^{-} 19\right.$ meters), or developing a macroscopic velocity field (screening currents), generating a macroscopic force field, compressing the elementary particles (the sources of these fields) into compact astronomical bodies. This is a macroscopic manifestation of the Higgs mechanism, which is related with the gravitational force fields.

In superconductors, stationary circulation fields of the SCC along closed loops (local phase or local Goldstone modes) are caused by the vector potential field associated with magnetic field. In order to reduce energy, the superconductor confines the magnetic field into quantized magnetic fluxons $\Phi_{o}$ involving them by circulation fields of the SCC along a closed loop (quantized Abrikosov vortices). This flow has a locked-in phase displacement $\theta$ along the loop:

$$
\theta=\frac{2}{\pi} \Phi_{o} \oint \vec{A} \cdot d \vec{l}=n 2 \pi
$$

In this equation $\vec{A}$ is the solenoidal vector potential, associated with the magnetic field and $d \vec{l}$ is an infinitesimal vector line element along the closed integration path. Single valuedness requires that the total phase displacements $\theta$ round the loops be an integer multiple of $2 \pi$. Equation (2) rules the intrinsic quantization of excitations in quantum fluids. This intrinsic quantization is due to spatial confinement of the excitation, however the origin of this confinement is completely different from that of a usual particle confined by a potential well. Visibly this confinement is ruled by minimization of energy. Displacing the phase over a larger volume of the condensate cost more energy than over a smaller one. The very stable Rotons, Maxons and Vortices in superfluids and Abrikosov vortices in superconductors are examples of such intrinsically quantized quasi-particles. In the $\mathrm{HC}$, an analogous intrinsic quantization mechanism must be responsible for the quantization of all the different parameters associated with the elementary particles.

\section{Experimental Observations and Theoretical Support Showing that the Accelerated Expansion of the Universe is Accelerated Expansion of the Higgs Quantum Space Itself}

Although the true nature of dark energy is a mystery, its presence is actually an indubitable observational fact. Nevertheless, other well-known experimental and observational facts certainly may give a clue about the possible nature of dark energy or even may fully uncover it. As affirmed in the introduction, the consensus of the cosmologists is that dark energy is not usual mass-energy, but is energy of space itself and distributes it very homogeneously throughout the universe. Therefore, in spite of its total amount being much larger than that of the ordinary mass-energy, its density is low (about $7 \times 10^{-27} \mathrm{~kg} / \mathrm{m}^{3}$ ). It also is consensus that dark energy interacts with ordinary mass-energy only by gravity. All these features arise from plausibility considerations from cosmological observations. These assumptions however are made without specifying their exact physical origin. [12] The nature of dark energy thus remains unknown.

The present work will show that all these features of dark energy arise naturally within the scenario of the Higgs Quantum Space (HQS) dynamics, stemming from the physics creating the universe. Condensation of the Higgs bosons liberates vast amounts of energy, in the order of $120 \mathrm{GeV}$ per Higgs boson. However, the energy of the Higgs condensate (HC) cannot fall directly to the minimum of energy, because of lack of efficient dissipation mechanisms. During condensation a persistent Higgs mode may be excited, involving oscillations of the density $\left(\rho=\phi^{*} \phi\right)$ and of the global volume of the HQS. If $\rho>n / m$, the HC can lower its energy by expanding its volume and if $\rho<n / m$, it can lower its energy by shrinking its volume. Contraction or expansion of the volume of the HC involves vast amounts of energy. On the other hand, the presence of the ordinary mass-energy in the universe represents a persistent phase disorder that holds back the advance toward the minimum of energy. The HC however can gradually lower its energy by confining and compressing this ordinary mass-energy into compact astronomical bodies by gravity. Confinement of ordinary mass-energy and global volumetric expansion of the HQS are mechanisms by which the HC can dissipate and lower its energy. This provides strong evidence that the condensation process of the $\mathrm{HC}$ still is actually in course.

\subsection{The Experimental Evidence}

According to the Higgs theory, [13, 14, 15] the Higgs Quantum Space (HQS) rules the inertial motion of matter and propagates light. Several recent experimental observations, $[6,7,16]$ achieved with the help of the tightly synchronized clocks in orbit, demonstrate that this HQS is not static, 
but is circulating round earth and round the sun according to a velocity field consistent with the local main astronomical motions. The tightly synchronized clocks in the satellites of the GRACE project, moving at nearly $8 \mathrm{~km} / \mathrm{sec}$ in a same polar orbit, allowed for precise measurements of the one-way velocity of the electromagnetic signals (light) between these satellites, in both senses. They have clearly demonstrated that the signals have a backward anisotropy of exactly the orbital velocity of the satellites.[16] These observations irremediably break by the first time the century old believe of the intrinsic constancy and isotropy of light with respect to all possible inertial references. This result demands for an immediate reconsideration of the interpretation of all the light anisotropy experiments performed in the past century. Most of the light anisotropy experiments searched for effects of the orbital and cosmic motion of earth and all obtained closely null results. These observations show unambiguously that earth is very closely stationary with respect to the HQS propagating light, which is possible only if the HQS is moving round the sun and round the galactic center in consonance with the earth's and the solar system's orbital motions.[8]

Another, even much more important experimental observation is the absence of gravitational time dilation effects of the solar gravitational potential on the GPS clocks. [6, 7] This gravitational time dilation is predicted by the General Theory of Relativity but not observed. It proves that it is not the gravitational potential that causes the gravitational time dilation, however the velocity with respect to the HQS.[8] In particular, the GPS clocks follow the orbital motion of earth in the solar Keplerian velocity field of the HQS, creating the solar gravitational field and hence are stationary with respect to this HQS. In fact all the planets must be very closely stationary with respect to the HQS in the solar field. In terms of the usual spherical coordinates $[r, \theta, \phi]$, this Keplerian velocity field has the form:

$$
\left(\vec{V}(r)=[\gamma M / r]^{1 / 2} \vec{e}_{\phi}\right.
$$

where $\gamma$ is the Newtonian gravitational constant, $M$ is the source mass, $r$ is the radial coordinate and $\vec{e}_{\phi}$ is a unit vector along the azimuthal spherical coordinate. This is a cylindrical velocity field the magnitude of the velocity being spherically symmetric. This Keplerian velocity field of the HQS, ruling the inertial motion of matter-energy, appropriately creates the gravitational dynamics, observed on earth and in the solar system. [8, 17, 18, 19] An analogous velocity field of the HQS creates the observed non-Keplerian gravitational dynamics within the galaxies, without the need of dark matter.[20]

The slowing of clocks, moving in free space, is caused by their velocity with respect to the HQS. The gravitational slowing of clocks, stationary with respect to ordinary space coordinates within the Keplerian velocity field creating a gravitational field, is due to the velocity of the HQS through the stationary clocks (implicit velocity of clocks with respect to the HQS). For a complete but succinct description of all these conclusions please see Ref. [8]

In the velocity fields round the sun and round the galaxy, earth is very closely stationary with respect to the HQS, which directly explains the null results of the Michelson light anisotropy experiments. It also explains in terms of simple and well-known physics all the effectively observed effects, caused by the gravitational fields on the propagation of light and on the rate of clocks.[8] It, in particular, explains the one-way anisotropy of light, recently measured very precisely with the help of the twin satellites of the GRACE project along a same polar orbit. The Keplerian velocity field of earth has no component along the polar orbit and hence the one-way anisotropy is due exclusively to the velocity of the GRACE satellites. The solar Keplerian velocity field of the HQS also explains the absence of the gravitational time dilation, due to the solar gravitational potential on the GPS clocks, which is predicted by General Relativity but is not observed.[6, 7] According to the theory of the HQSdynamics, slowing of the clock rates is due to exclusively to velocity with respect to the HQS. [8] The absence of clock slowing in the GPS satellites is simply due to the fact that earth together with the GPS satellites are commoving with the HQS in the solar Keplerian velocity field and hence are stationary with respect to the HQS. According to the theory of the HQS-dynamics, the solar system also is comoving with the HQS round the galactic center.[8] The null results of the Michelson light anisotropy experiments and absence of slowing of the GPS clocks, due to the solar gravitational field, provide clear evidence that the so called relativistic effects are not due to relative velocity with respect to the observer, however to velocity with respect to the HQS.[20]

While the local HQS-dynamics round the sun and round the galaxy properly explains the isotropy of light with respect to earth, it cannot explain why the recession of the galaxies causes no light anisotropy. Within this HQS-dynamics scenario, an appropriate explanation of the accelerated expansion of the universe can of course not run into conflict with the null results of the Michelson light anisotropy experiments. The fact that the velocity of light is isotropic with respect to earth, despite its motion in the solar system, in the Milky-Way galaxy and also in spite of the recession of the galaxies, demonstrates that the accelerated expansion of the universe does not set these astronomical bodies into motion with respect to the HQS. Despite the accelerated expansion of the universe, our Milky-Way galaxy remains stationary with respect to the local HQS. It certainly would not be reasonable to assume that our galaxy is in a privileged kinematic circumstance with respect to the HQS in detriment to all the remainder galaxies in the universe. All the galaxies must equally be closely resting with respect to the HQS. This inexorably leads to the conclusion that the HQS, ruling the inertial motion of matter and propagating light, besides moving according to velocity fields, consistently with the local main astronomical motions, also is expanding consistently with the recession of the galaxies. In this case the Hubble spectral red-shift of light from distant galaxies, is of course not a usual Doppler shift, but is due to a time-rate of stretching of the wavelength, due to expansion of the HQS, its medium of propagation. It is easy to show that this stretching, as a function of time, causes exactly the same red shift as the usual Doppler shift, due to conventional recession. It also stretches the wavelength of particles, thereby reducing their velocity with respect to the HQS.

\subsection{Theoretical Support}

The only conceivable physical mechanism, able to produce enough energy to drive the observed accelerated expansion of the universe, can be found in the scenario of the creation of the universe. The energy of the Higgs condensate (HC) within the Higgs potential energy well (Fig.1) depends on the condensate density $\rho=\phi^{*} \phi$. The Higgs potential energy 
well is generic to all positions in space throughout the universe and thus is a universal field of potential energy. The variation of the potential energy of the $\mathrm{HC}$, due to variation of the spatial density $\rho$ and related with volumetric expansion of the $\mathrm{HC}$ in a Higgs mode can easily achieve amounts of energy in the order of magnitude of dark energy. This energy obviously is not usual mass-energy, but is energy of space (HQS) itself. Moreover, the density of the HC, while a quantum condensate, necessarily is very homogeneous throughout space and the depth of the Higgs potential energy well is very closely constant (flat) throughout the universe. This means that dark energy (associated with the HQS) intrinsically is distributed homogeneously throughout space as conjectured by cosmologists. This is the reason why its density is very low $\left(\rho \sim 7 \times 10^{-27} \mathrm{~kg} / \mathrm{m}^{3}\right)$.

If $\rho>n / m$, the energy of the $\mathrm{HC}$ is not minimum. It however can lower it by volumetric expansion. As the deepness of the Higgs potential well is very flat, the expansion of the HQS has no barriers and hence the rate of expansion is accelerated. Moreover, the only relationship of the HQS with ordinary mass-energy is providing inertial mass to the elementary particles, which means ruling the inertial motion of matter-energy. Hence, the only way to the HQS-dynamics to affect the motion of ordinary matter is by inertial effects, which means gravitational effects. If the HQS were static or moving likewise a rigid body, the motions of all free particles and light would, in principle, be along straight lines (no gravitation). If however the HQS moves non-uniformly, it necessarily induces an inertial dynamics. On moving round astronomical bodies according to a Keplerian velocity field (Eq.(3)), consistent with the local main astronomical motions, it generates the gravitational dynamics as observed in the gravitational fields.[8]. These features of the HQS fully and consistently meet all the features of dark energy, exactly as conceived in cosmology. Moreover, they all hint consistently to the conclusion that the accelerated expansion of the HQS is the drive of the accelerated expansion of the universe.

In practice it is possible to imagine that, shortly after the Big-Bang, the density $\rho=\phi^{*} \phi$ of the Higgs condensate (HC) certainly was lower than the equilibrium density $n / m$. In this stage, the HC could lower its energy by accelerated volumetric contraction, possibly with formation of droplets, analogously as fluctuations in superconductivity during the superconducting transition. However, at a given stage, $\rho$ must have achieved the equilibrium value, after which decelerating contraction may have driven the density $\rho$ to a maximum, on from which an accelerated expansion must have started. This sequence of events must have triggered a Higgs mode of the HQS. Astronomical observations show that actually the universe is in a stage of accelerated expansion. If the HQS is conservative likewise superfluids and the SCC, then this Higgs mode oscillations may be persistent. Moreover, besides this, the HC must actually still be struggling toward the minimum of energy against the persistent phase disorder, due to the usual mass-energy in the universe.

In summary, the HQS, ruling the inertial motion of matter and the propagation of light, is circulating round earth, round the sun and round the galactic center, consistently with the local main astronomical motions, thereby creating the observed gravitational dynamics. This is a macroscopic manifestation of the Higgs mechanism, confining the matter fields. In this local HQS-dynamics round the sun and round the galactic center, earth is very closely stationary with respect to the local HQS, so that the orbital motion of earth and the motion of the solar system within the galaxy, cause no light anisotropy with respect to earth. Moreover, the accelerated expansion of the universe is consistent with the accelerated expansion of the HQS itself, in this way too letting earth stationary with respect to the local HQS. This concomitant expansion ultimately explains the observed isotropy of light with respect to earth.

\section{Conclusions}

According to the present work, the isotropy of light with respect to earth proves that the accelerated expansion of the universe necessarily must be concurrent with the accelerated expansion of the Higgs quantum space itself. On the other hand the amount of energy, liberated during the Higgs mode oscillations, due to variations of its density $\rho=\phi^{*} \phi$ and of its volume, is within the order of magnitude of the estimates of dark energy and thus can drive the observed accelerated expansion of the universe. This scenario of the HQS-dynamics, besides creating the observed gravitational dynamics, [8] appropriately reproduces all the features of dark energy, exactly as ascertained by the cosmologists. Namely that the amount of dark energy is much larger than the total ordinary massenergy in the universe, that it is not usual mass-energy, but is energy of space itself, that it distributes it homogeneously throughout the universe and that it interacts with ordinary mass-energy only by gravity.

\section{REFERENCES}

[1] A. Riess et al., "Observational evidence from supernovae for an accelerating universe and a cosmological constant", Astronomical Journal, 116116, 1009 (1998).

[2] S. Perlmutter et al., "Measurements of $\Omega$ and $\Lambda$ from 42 High-Redshift Supernovae", Astrophysical Journal, 517517, 565 (1999).

[3] F.S.N. Lobo, "Stable dark energy stars," Class. Quant. Grav., 23, 1525 (2006).

[4] F.S.N. Lobo, "Stability of phantom wormholes," Phys. Rev. D71, 043520 (2005).

[5] M.Malaver and M. Esculpi, "A theoretical model of stable dark enrgy stars," IJRRAS, 14 (1), 26-39 (2013).

[6] R.R. Hatch, "Those Scandalous Clocks", GPS Solutions 8, 67 (2004).

[7] R.R. Hatch, "Clocks and the Equivalence Principle", Foundations of Physics, 34 (11), 1725 (2004).

[8] J. Schaf, "The Higgs Quantum Space Dynamics Generating the Gavitational Dynamics in the Universe", Universal Journal of Physics and Applications" 9141 (2015).

[9] P.W. Anderson, "Plasmons, Gauge Invariance, and Mass." Phys. Rev. 130, 439 (1963). 
[10] W. Meissner and R. Ochsenfeld, "Ein neuer Effekt bei Eintritt der Supraleitfaehigkeit." Naturwissenschaften 21, 787 (1933).

[11] F.T. Dias, P. Pureur, P. Rodrigues Jr, and X. Obradors, "Paramagnetic effect at low and high magnetic fields in melt-textured $Y B a 2 C u 3 O 7-\delta$ ", Phys. Rev. B 70, 224519 (2004).

[12] R. Caldwell, M. Kamionkowski, Nature 458, 587-589 (2 April 2009).

[13] P. W. Higgs, "Broken Symmetries and the Masses of Gauge Bosons", Physical Review Letters 13 (16), 508 (1964).

[14] F. Englert and R. Brout, "Broken symmetry and the mass of gauge vector mesons" Phys. Rev. Lett. 13, 321323, (1964).

[15] T. W. B. Kibble, "Englert-Brout-Higgs-GuralnikHagen-Kibble mechanism", Scholarpedia, 4 (1), 8741, (2009).
[16] R.R. Hatch, "New Theory of Gravity: Overcoming Problems with General Relativity", Physics Essays 20, 83 (2007).

[17] J. Schaf, "The Nature of Space and of the Gravitational Physics in the Light of Recent New Experimental Observations and Within the Scenario of the Higgs Mechanism." Recent Progress in Space Technology, 4, 44-66 (2014).

[18] J. Schaf, "Gravitational Fields: Another Fortunate Manifestation of the Higgs Mechanism," Journal of Modern Physics, 5, 407-448 (2014).

[19] J. Schaf, "The Nature of Space and of the Gravitational Fields Revealed by Recent Experimental Observations and Also Implicit in the Quantum Field Theory", Journal of Modern Physics, 4, 676-685, (2013).

[20] J. Schaf, "The Fundamental Assumptions of the Theory of Relativity Shown False, Yet Many Predictions Match Observations.This Work Shows Why." Journal of Modern Physics, 5, 1617-1639 (2014). 